PROCEEDINGS OF THE

AMERICAN MATHEMATICAL SOCIETY

Volume 137, Number 9, September 2009, Pages 3067-3075

S 0002-9939(09)09837-2

Article electronically published on February 19, 2009

\title{
POSITIVE AND NEGATIVE RESULTS ON THE NUMERICAL INDEX OF BANACH SPACES AND DUALITY
}

\author{
MIGUEL MARTÍN \\ (Communicated by Nigel J. Kalton)
}

\begin{abstract}
We show that the numerical index of an $L$-embedded space and that of its dual coincide. In particular, the numerical index of the predual of a real or complex von Neumann algebra or $J B W^{*}$-triple coincides with the numerical index of the space. Also, we prove that when $X$ is an $M$ embedded Banach space with numerical index 1, then every closed subspace of $X^{* *}$ containing $X$ also has numerical index 1 (in particular, $X^{*}$ and $X^{* *}$ have numerical index 1). Finally, we show that any Banach space $X$ containing a complemented copy of $c_{0}$ or a copy of $\ell_{\infty}$ admits an equivalent norm for which the numerical index of its dual space is strictly less than the index of the space. In the special case of a separable space $X$ containing $c_{0}$, it is actually possible to renorm $X$ with the maximum value of the numerical index (namely 1 ) while the numerical index of the dual is as small as possible (namely, 0 in the real case, $1 / \mathrm{e}$ in the complex case).
\end{abstract}

\section{INTRODUCTION}

The concept of the numerical index of a Banach space was first suggested by G. Lumer in 1968 (see [10]), and it is a parameter relating the norm and the numerical range of operators on the space. Let us recall the necessary definitions and notation. For a real or complex Banach space $X$, we write $B_{X}$ for the closed unit ball and $S_{X}$ for the unit sphere of $X$. The dual space is denoted by $X^{*}$, and the Banach algebra of all bounded linear operators on $X$ by $L(X)$. The numerical range of an operator $T \in L(X)$ is the subset $V(T)$ of the scalar field given by

$$
V(T):=\left\{x^{*}(T x): x \in S_{X}, x^{*} \in S_{X^{*}}, x^{*}(x)=1\right\} .
$$

The numerical radius of $T$ is the seminorm defined on $L(X)$ by

$$
v(T):=\sup \{|\lambda|: \lambda \in V(T)\}
$$

for each $T \in L(X)$. The numerical index of the space $X$ is the constant $n(X)$ defined by

$$
n(X):=\inf \{v(T): T \in L(X),\|T\|=1\}
$$

Received by the editors August 6, 2008, and, in revised form, November 20, 2008.

2000 Mathematics Subject Classification. Primary 46B20, 46B04, 47A12.

Key words and phrases. Numerical range, numerical index, duality, $L$-embedded, $M$-embedded.

The author was supported by Spanish MEC project MTM2006-04837 and Junta de Andalucía grants FQM-185 and FQM-1438.

(C)2009 American Mathematical Society Reverts to public domain 28 years from publication 
or, equivalently, the greatest constant $k \geqslant 0$ such that $k\|T\| \leqslant v(T)$ for every $T \in L(X)$. Observe that, clearly, $0 \leqslant n(X) \leqslant 1$ for every Banach space $X$. Classical references here are the monographs by F. Bonsall and J. Duncan [6, 7] from the 1970's. The reader will find the state-of-the-art on numerical index in the recent survey paper [15, to which we refer for background.

Let us mention here a couple of facts which will be relevant to our discussion. First, the set of possible values of the numerical index is as follows [10]:

$$
\begin{aligned}
\{n(X): X \text { complex Banach space }\} & =\left[\mathrm{e}^{-1}, 1\right], \\
\{n(X): X \text { real Banach space }\} & =[0,1] .
\end{aligned}
$$

Given a bounded linear operator $T$ on a Banach space $X$, it is a well-known fact in the theory of numerical ranges (see [6, $\S 9]$ ) that

$$
\sup \operatorname{Re} V(T)=\lim _{\alpha \downarrow 0} \frac{\|\operatorname{Id}+\alpha T\|-1}{\alpha} \quad \text { and so } \quad v(T)=\max _{|\omega|=1} \lim _{\alpha \downarrow 0} \frac{\|\operatorname{Id}+\alpha \omega T\|-1}{\alpha} .
$$

It is immediate from the above formula that $v(T)=v\left(T^{*}\right)$, where $T^{*} \in L\left(X^{*}\right)$ is the adjoint operator of $T$, and the result given in [10, Proposition 1.3] that

$$
n\left(X^{*}\right) \leqslant n(X)
$$

clearly follows.

The question if the above inequality is actually an equality had been around from the beginning of the subject. It was solved in the negative in 2007, when K. Boyko, V. Kadets, M. Martín, and D. Werner [8, Example 3.1] found an example of a Banach space whose numerical index is strictly greater than the numerical index of its dual. Namely, the Banach space

$$
X=\left\{(x, y, z) \in c \oplus_{\infty} c \oplus_{\infty} c: \lim x+\lim y+\lim z=0\right\}
$$

satisfies that $n(X)=1$ and $n\left(X^{*}\right)<1$ in both the real and the complex case. Actually, essentially with the same techniques, it is shown in [8. Examples 3.3] that there are examples of Banach spaces $Y_{1}$ in the real case and $Y_{2}$ in the complex case, both isomorphic to $c_{0}$, such that

$$
n\left(Y_{1}\right)=1, \quad n\left(Y_{1}^{*}\right)=0, \quad \text { and } \quad n\left(Y_{2}\right)=1, \quad n\left(Y_{2}^{*}\right)=1 / \mathrm{e} .
$$

There are some particular cases in which the equality in (1) holds. Namely, it is clear that $n(X)=n\left(X^{*}\right)$ for every reflexive space $X$, and this equality also holds whenever $n\left(X^{*}\right)=1$, in particular when $X$ is an $L$ - or an $M$-space. It is also true that $n(X)=n\left(X^{*}\right)$ when $X$ is a $C^{*}$-algebra or a von Neumann algebra predual (see [17, Remark 2.7]).

Let us comment that all the nontrivial results above are true by computation: the numerical index of a (complex) $C^{*}$-algebra $X$ is 1 or $1 / 2$ depending on the fact that $X$ is commutative or not, and when $X$ is a (complex) von Neumann algebra, the numerical index of its unique predual is 1 or $1 / 2$ depending also on the commutativity of the algebra.

This paper deals with the question of whether (1) is an equality in some particular cases. First, we give a new positive result which does not depend on the computation of the numerical indices of the spaces. Namely, we show that the equality in equation (11) holds for $L$-embedded spaces. Examples of $L$-embedded spaces are the reflexive ones (trivial) and the preduals of von Neumann algebras, so our result gives a nonalgebraic proof of the aforementioned result of [17]. Moreover, we can prove that actually the equality in equation (1) holds for the larger class of 
those preduals of real or complex $J B W^{*}$-triples since they are $L$-embedded. Let us also comment that the computation of the numerical index of real or complex $J B W^{*}$-triples is not achieved yet. All this is shown in section 2 ,

Another already-known case in which the equality in inequality (11) holds is when $X$ has the Radon-Nikodým property and $n(X)=1$ [8, Proposition 4.1]. We prove a result along these lines by showing that the dual of an $M$-embedded space with numerical index 1 also has numerical index 1 . Actually, we show that if $X$ is an $M$-embedded space with $n(X)=1$, and $Y$ is a subspace of $X^{* *}$ containing $X$, then $n(Y)=1$. This is the content of section 3 .

Finally, in section 4 we will show that any Banach space containing an isomorphic complemented copy of $c_{0}$ or an isomorphic copy of $\ell_{\infty}$ admits an equivalent norm for which inequality (1D) is strict. If we actually have a separable Banach space containing an isomorphic copy of $c_{0}$, then it is possible to renorm the space such that the numerical index of the renormed space is 1 , while the numerical index of its dual is as small as possible. In particular, this happens for separable nonreflexive $M$-embedded spaces.

\section{2. $L$-EMBEDDED SPACES}

We recall that a Banach space $X$ is said to be $L$-embedded if $X^{* *}=X \oplus_{1} X_{s}$ for some closed subspace $X_{s}$ of $X^{* *}$. We refer to [13] for background. Examples of $L$-embedded spaces are the reflexive ones (trivial), preduals of von Neumann algebras (in particular, $L_{1}(\mu)$ spaces), the Lorentz spaces $d(w, 1)$ and $L^{p, 1}$ (see [13, Examples III.1.4 and IV.1.1]). As claimed in the introduction, we show that the numerical index of an $L$-embedded space and that of its dual coincide.

Theorem 2.1. Let $X$ be an L-embedded space. Then, $n(X)=n\left(X^{*}\right)$.

Proof. We write $X^{* *}=X \oplus_{1} X_{s}$ and $P_{X}: X^{* *} \longrightarrow X$ for the associate projection. Given $T \in L\left(X^{*}\right)$, we consider the operators $A \in L(X)$ and $B \in L\left(X, X_{s}\right)$ given by $A=P_{X} \circ T^{*} \circ i_{X}$ and $B=\left(\operatorname{Id}-P_{X}\right) \circ T^{*} \circ i_{X}$ (observe that $\left.T^{*} \circ i_{X} \equiv A \oplus B\right)$. Given $\varepsilon>0$, since $B_{X}$ is $w^{*}$-dense in $B_{X^{* *}}$ and $T^{*}$ is $w^{*}$-w*-continuous, we may find $x_{0} \in S_{X}$ such that

$$
\left\|T^{*}\left(x_{0}\right)\right\|=\left\|A\left(x_{0}\right)\right\|+\left\|B\left(x_{0}\right)\right\|>\left\|T^{*}\right\|-\varepsilon .
$$

Now, we find $a_{0} \in S_{X}, y_{0}^{*} \in S_{X_{s}^{*}}$ satisfying

$$
\left\|A\left(x_{0}\right)\right\| a_{0}=A\left(x_{0}\right) \quad \text { and } \quad y_{0}^{*}\left(B\left(x_{0}\right)\right)=\left\|B x_{0}\right\|,
$$

and we define an operator $S \in L(X)$ by

$$
S(x)=A(x)+y_{0}^{*}(B(x)) a_{0} \quad(x \in X) .
$$

Then

$$
\|S\| \geqslant\left\|S\left(x_{0}\right)\right\|=\left\|A\left(x_{0}\right)+\right\| B\left(x_{0}\right)\left\|a_{0}\right\|=\left(\left\|A x_{0}\right\|+\left\|B\left(x_{0}\right)\right\|\right)\left\|a_{0}\right\|>\left\|T^{*}\right\|-\varepsilon,
$$

so we may find $x \in X, x^{*} \in X^{*}$ such that

$$
\|x\|=\left\|x^{*}\right\|=x^{*}(x)=1 \quad \text { and } \quad\left|x^{*}(S(x))\right| \geqslant n(X)[\|T\|-\varepsilon] .
$$

For $z=(x, 0) \in S_{X^{* *}}$ and $z^{*}=\left(x^{*}, x^{*}\left(a_{0}\right) y_{0}^{*}\right) \in S_{X^{* * *}}$ we clearly have $z^{*}(z)=1$ and

$$
\left|z^{*}\left(T^{*}(z)\right)\right|=\left|x^{*}(A(x))+x^{*}\left(a_{0}\right) y_{0}^{*}(B(x))\right|=\left|x^{*}(S(x))\right| \geqslant n(X)\left[\left\|T^{*}\right\|-\varepsilon\right] .
$$


Now, letting $\varepsilon \downarrow 0$, we get

$$
v(T)=v\left(T^{*}\right) \geqslant n(X)\left\|T^{*}\right\|=n(X)\|T\| .
$$

Therefore, $n\left(X^{*}\right) \geqslant n(X)$, and the other inequality is always true. Finally, let us say that this proof is based on that of [21, Proposition 1].

Let us comment that it has been shown recently that separable $L$-embedded Banach spaces are unique preduals of their duals 22. Therefore, it makes sense to ask whether a Banach space $X$ having a unique predual $X_{*}$ satisfies $n\left(X_{*}\right)=n(X)$.

Since preduals of von Neumann algebras are $L$-embedded, the above result gives a nonalgebraic proof of the aforementioned result of [17 that the numerical index of a von Neumann algebra and that of the algebra coincide. Moreover, the main application of the above result is obtained when one deals with the so-called preduals of $J B W^{*}$-triples. We recall that a $J B W^{*}$-triple is a $J B^{*}$-triple which is a dual Banach space, in which case it has a unique predual. Complex $J B^{*}$-triples are those complex Banach spaces whose open unit balls are homogeneous with respect to biholomorphic transformations, and real $J B^{*}$-triples are the real forms of complex $J B^{*}$-triples. We refer the reader to [12, 14, 23, for background. As we already commented in the introduction, preduals of real or complex $J B W^{*}$-triples are $L$-embedded ([12] for the complex case and 4 for the real case), so the following result follows. In the complex case, this was already known for $J B^{*}$-algebras 17 , Remark 2.7]. Let us comment that the computation of the numerical index of real $C^{*}$ algebras and real or complex $J B^{*}$-triples has not been achieved yet.

Corollary 2.2. Let $X$ be a real or complex $J B W^{*}$-triple and let $X_{*}$ be its (unique) predual. Then, $n\left(X_{*}\right)=n(X)$. In particular, this happens when $X$ is a real or complex von Neumann algebra.

We finish this section with a result for the so-called Daugavet property and alternative Daugavet property. We recall that a Banach space $X$ has the Daugavet property if every rank-one operator $T \in L(X)$ satisfies the Daugavet equation

$$
\|\operatorname{Id}+T\|=1+\|T\| .
$$

In such a case, all weakly compact operators on $X$ also satisfy (DE). A Banach space $X$ is said to have the alternative Daugavet equation if every rank-one operator $T \in L(X)$ satisfies the equality

$(\mathrm{aDE})$

$$
\max _{|\omega|=1}\|\operatorname{Id}+\omega T\|=1+\|T\| ;
$$

i.e. for every rank-one operator $T$ there is a modulus-one scalar $\omega$ such that $\omega T$ satisfies (DE). Analogously to the situation for the Daugavet property, if a Banach space $X$ has the alternative Daugavet property, then all weakly compact operators in $L(X)$ also satisfy (aDE). Good references for the Daugavet property are the books [1, 2] and the papers [16, 24. Information on the alternative Daugavet property can be found in [19, 20].

It is clear that both the Daugavet property and the alternative Daugavet property pass from the dual of a Banach space to the space, but the converse result does not always hold (consider $C\left([0,1], \ell_{2}\right)$ ). Our next aim is to adapt the proof of Theorem 2.1 to show that such converses hold for $L$-embedded spaces. To do so, we need just two observations. First, we make use of an old result from the 1970's 10. saying that an operator $T \in L(X)$ satisfies (DE) if and only if $\sup \operatorname{Re} V(T)=\|T\|$, 
and so $T$ satisfies (aDE) if and only if $v(T)=\|T\|$. Second, we take into account that in the proof of Theorem 2.1. if one starts with a rank-one operator, then all operators involved are finite-rank and that we may write (4) in terms of real parts instead of moduli.

Proposition 2.3. Let $X$ be an L-embedded Banach space.

(a) If $X$ has the Daugavet property, then so does $X^{*}$.

(b) If $X$ has the alternative Daugavet property, then so does $X^{*}$.

For the Daugavet property, the above result was previously known for preduals of real or complex $J B W^{*}$-triples (see [5, Theorem 3.2]). In the case of the alternative Daugavet property, we knew the result only for complex $J B W^{*}$-triples (see [20, Theorem 4.6] or [19, Theorem 2.3]).

\section{3. $M$-EMBEDDED SPACES}

We recall that a Banach space $X$ is $M$-embedded if $X^{\perp}$ is an $L$-summand of $X^{* * *}$ or, equivalently, if the natural (Dixmier) projection from $X^{* * *}$ onto $X^{*}$ is an $L$-projection (i.e. $\left.X^{* * *}=i_{X^{*}}\left(X^{*}\right) \oplus_{1} X^{\perp}\right)$. We refer the reader to [13] for more information and background. Typical examples of $M$-embedded spaces are $c_{0}(\Gamma)$ for any set $\Gamma$ and $K(H)$, the space of compact operators on a Hilbert space $H$ [13. Examples III.1.4].

The following is another particular case in which the equality in (1) holds.

Proposition 3.1. Let $X$ be an $M$-embedded space. If $n(X)=1$, then

$$
n\left(X^{*}\right)=n\left(X^{* *}\right)=1 \text {. }
$$

Proof. Let $A$ be the set of all $w^{*}$-strongly exposed points of $B_{X^{*}}$. By [13, Corollary III.3.2] we have

$$
B_{X^{*}}=\overline{\mathrm{co}}(A)
$$

(norm closure). On the other hand, [18, Lemma 1] gives us that

$$
\left|x^{* *}\left(a^{*}\right)\right|=1 \quad\left(x^{* *} \in \operatorname{ext}\left(B_{X^{* *}}\right), a^{*} \in A\right),
$$

where $\operatorname{ext}\left(B_{X^{* *}}\right)$ holds for the set of extreme points of $B_{X^{* *}}$. This easily implies that $n\left(X^{*}\right)=1$ (see [15, Proposition 6.a]). Now, $X^{*}$ is $L$-embedded by [13, Corollary III.1.3], and thus Theorem 2.1 gives $n\left(X^{* *}\right)=n\left(X^{*}\right)=1$.

\section{Remarks 3.2.}

(a) If $X$ is an $M$-embedded space, then $X^{*}$ is $L$-embedded [13, Corollary III.1.3]. We may wonder if Proposition 3.1 can be extended to any Banach space whose dual is $L$-embedded. But this is not the case. For instance, the dual of the space $X$ given in equation (2) is $L$-embedded, $n(X)=1$, but $n\left(X^{*}\right)<1$. See [8, Example 3.1] for the details.

(b) If $X$ is an $M$-embedded space, then it is Asplund [13, Theorem III.3.1]. We may wonder if Proposition 3.1 can be extended to any Asplund space. This is not the case, as shown in the same example of item (a).

The proof given for Proposition 3.1 can actually be extended to get the following more general result. We decided to write two different statements for the sake of clarity. 
Theorem 3.3. Let $X$ be an $M$-embedded space with $n(X)=1$. If $Y$ is a closed subspace of $X^{* *}$ containing (the canonical copy of) $X$, then $n(Y)=1$.

Proof. Since we have $X \subset Y \subset X^{* *}$ and $X$ is an $M$-ideal of $X^{* *}$, we deduce that $X$ is an $M$-ideal of $Y$ [13, Proposition I.1.17], meaning that $X^{\perp}$ is an $L$-summand of $Y^{*}$. By [13, Remark 1.13], we get that

$$
Y^{*} \equiv X^{*} \oplus_{1} X^{\perp} \quad \text { and so } \quad Y^{* *} \equiv X^{* *} \oplus_{\infty}\left[X^{\perp}\right]^{*} .
$$

Now, as we did in the proof of Proposition 3.1, we consider the set $A$ of those $w^{*}$-strongly exposed points of $B_{X^{*}}$, and so we write

$$
\mathcal{A}=\left\{\left(a^{*}, 0\right) \in S_{Y^{*}}: a^{*} \in A\right\} ;
$$

i.e. $\mathcal{A}$ is the restriction to $Y$ of the canonical image of $A \subset X^{*}$ in $X^{* * *}$.

On the one hand, for every $y \in Y$ we have

$$
\|y\|=\sup \left\{\left|y^{*}(y)\right|: y^{*} \in \mathcal{A}\right\}
$$

(this is an immediate consequence of (5) and the fact that $Y \subset X^{* *}$ ), and this implies that

$$
B_{Y^{*}}=\overline{\mathrm{co}} w^{*}(\mathcal{A}) .
$$

On the other hand, since $Y^{* *}=X^{* *} \oplus_{\infty}\left[X^{\perp}\right]^{*}$, the set of extreme points of $B_{Y^{* *}}$ can be described as

$$
\operatorname{ext}\left(B_{Y^{* *}}\right)=\left\{\left(x^{* *}, \xi\right): x^{* *} \in \operatorname{ext}\left(B_{X^{* *}}\right), \xi \in \operatorname{ext}\left(B_{\left[X^{\perp}\right]^{*}}\right)\right\} .
$$

Now, we get from (6) that

$$
\left|y^{* *}\left(y^{*}\right)\right|=1 \quad\left(y^{* *} \in \operatorname{ext}\left(B_{Y^{* *}}\right), y^{*} \in \mathcal{A}\right) .
$$

This fact, together with (7), gives us $n(Y)=1$ (see [15, Proposition 6.c] or [19, Lemma 1.1]).

Remarks 3.4.

(a) As we already commented, a typical example of an $M$-embedded space is $c_{0}(\Gamma)$ for any set $\Gamma$. In this case, the above proposition says that a closed subspace $Y$ of $\ell_{\infty}(\Gamma)$ containing $c_{0}(\Gamma)$ has numerical index 1. But this result was previously known, since in this case $Y$ has the so-called property $\beta$ with constant 0 , and this implies $n(Y)=1$ (see [11, Remark 8]).

(b) It is not always possible to get $n\left(Y^{*}\right)=1$ in Theorem 3.3. Indeed, let $X$ be the example given in equation (2). Then, one clearly has that

$$
c_{0}(\mathbb{N} \times \mathbb{N} \times \mathbb{N}) \subset X \subset \ell_{\infty}(\mathbb{N} \times \mathbb{N} \times \mathbb{N}),
$$

but $n\left(X^{*}\right)<1$.

We finish the section with two observations about the Daugavet property and the alternative Daugavet property for $M$-embedded spaces.

Remarks 3.5. We recall that an $M$-embedded space $X$ is an Asplund space [13, Theorem III.3.1]. Therefore,

(a) $X$ does not have the Daugavet property (see [24, Corollary 2.5]). 
(b) If $X$ has the alternative Daugavet property, then $n(X)=1$ (see [15, Proposition 12]). Then, $n\left(X^{*}\right)=n\left(X^{* *}\right)=1$ by Proposition 3.1, and so $X^{*}$ and $X^{* *}$ have the alternative Daugavet property. Moreover, using Theorem 3.3 . the same argument gives that any closed subspace $Y$ of $X^{* *}$ containing $X$ has the alternative Daugavet property.

\section{Renorming to FAIL the Equality of nUmerical InDEX}

Our final aim in this paper is to show that it is easy to construct examples of Banach spaces $X$ for which $n(X)>n\left(X^{*}\right)$.

Theorem 4.1. Let $X$ be a Banach space containing a complemented copy of $c_{0}$ or a copy of $\ell_{\infty}$. Then, there is a Banach space $Z$ isomorphic to $X$ such that $n\left(Z^{*}\right)<n(Z)$.

Proof. Let us start with the case of $c_{0}$. We write $X=Y \oplus W$ such that $Y$ is isomorphic to $c_{0}$. In the real case, we use [11, Theorem 9] to get a real Banach space $W_{1}$ isomorphic to $W$ with $n\left(W_{1}\right)>0$, and we take the space $Y_{1}$ of (3), which is isomorphic to $c_{0}$ and satisfies $n\left(Y_{1}\right)=1$ and $n\left(Y_{1}^{*}\right)=0$. Now, we write $Z=Y_{1} \oplus_{\infty} W_{1}$ and use [21, Proposition 1] to get that

$$
n(Z)=\min \left\{n\left(Y_{1}\right), n\left(W_{1}\right)\right\}>0 \quad \text { and } n\left(Z^{*}\right)=\min \left\{n\left(Y_{1}^{*}\right), n\left(W_{1}^{*}\right)\right\}=0 .
$$

The argument in the complex case is analogous. We find a complex Banach space $W_{2}$ isomorphic to $W$ with $n\left(W_{2}\right)>1$ /e from [11, Theorem 9]. We consider the complex Banach space $Y_{2}$ of (3) which is isomorphic to $c_{0}$ and satisfies $n\left(Y_{2}\right)=1$ and $n\left(Y_{2}^{*}\right)=1 /$ e. Now, writing $Z=Y_{2} \oplus_{\infty} W_{2}$, we finish as in the real case.

Finally, the proof for the $\ell_{\infty}$ case is again completely analogous, taking into account two facts: first, that $\ell_{\infty}$ is complemented in any superspace (see 3 . Proposition 2.5.2] for instance); second, that the construction of the spaces $Y_{1}$ and $Y_{2}$ of (3) given in [8, Examples 3.3] can be easily adapted to get another two spaces with the same properties but isomorphic to $\ell_{\infty}$.

Since a $c_{0}$-subspace of a separable space is complemented (Sobczyk's Theorem; see [3. Corollary 2.5.9] for instance) and since separable spaces containing $c_{0}$ can be renormed to have numerical index 1 [9, Corollary 3.6], we may improve the above result in the separable case.

Theorem 4.2. Let $X$ be a separable Banach space containing $c_{0}$. Then, there is a Banach space $Z$ isomorphic to $X$ such that $n(Z)=1$ and

$$
n\left(Z^{*}\right)=0 \text { in the real case, } n\left(Z^{*}\right)=1 / \text { e in the complex case. }
$$

Proof. The proof is just a refinement of the proof of Theorem 4.1. First of all, since $c_{0} \simeq c_{0} \oplus c_{0}$, we may write $X=Y \oplus W$, where $Y$ is isomorphic to $c_{0}$ and $W$ is a separable space containing an isomorphic copy of $c_{0}$. On the one hand, we take a space $\widetilde{Y}$ isomorphic to $c_{0}$ such that $n(\widetilde{Y})=1$ and $n\left(\widetilde{Y}^{*}\right)$ is 0 or $1 /$ e depending on whether we are in the real or in the complex case (just take $\tilde{Y}$ equal to $Y_{1}$ or $Y_{2}$ of (3)). On the other hand, since $W$ is separable and contains $c_{0}$, [9, Corollary 3.6] provides us with a Banach space $\widetilde{W}$ isomorphic to $W$ such that $n(\widetilde{W})=1$. Then, $X$ is isomorphic to $Z=\widetilde{Y} \oplus_{\infty} \widetilde{W}$ and, as in the proof of Theorem 4.1, we may use [21, Proposition 1] to get that $n(Z)=1$ and $n\left(Z^{*}\right)=0$ in the real case, $n\left(Z^{*}\right)=1 / \mathrm{e}$ in the complex case. 
Since any nonreflexive $M$-embedded Banach space contains a complemented copy of $c_{0}$ [13, Corollary III.4.7], the above result applies in this case. In particular, this happens to any infinite-dimensional closed subspace of $c_{0}$.

Corollary 4.3. Let $X$ be a separable nonreflexive $M$-embedded space. Then, there is a Banach space $Z$ isomorphic to $X$ such that $n(Z)=1$ and

$$
n\left(Z^{*}\right)=0 \text { in the real case, } n\left(Z^{*}\right)=1 / \mathrm{e} \text { in the complex case. }
$$

In particular, this is the case for infinite-dimensional closed subspaces of $c_{0}$.

We finish this section with an easy example showing that there is no value of the numerical index other than the minimum one for which the equality in equation (11) always holds.

\section{Example 4.4.}

(a) For every $t \in] 0,1]$ there is a real Banach space $X_{t}$ with $n\left(X_{t}\right)=t$ and $n\left(X_{t}^{*}\right)=0$.

(b) For every $t \in] 1 / \mathrm{e}, 1]$ there is a complex Banach space $X_{t}$ with $n\left(X_{t}\right)=t$ and $n\left(X_{t}^{*}\right)=1 / \mathrm{e}$.

Proof. Just take $X=Y_{1}$ in the real case or $X=Y_{2}$ in the complex case of the equality (3) and $Z_{t}$ a two-dimensional space with $n\left(Z_{t}\right)=n\left(Z_{t}^{*}\right)=t$. Then, the space $X_{t}=X \oplus_{1} Z_{t}$ fulfills the required condition.

It would be of interest to investigate isomorphic properties assuring that the numerical index of a Banach space and that of the dual coincide. In the separable case, a necessary condition is that no copy of $c_{0}$ is contained. We do not know if it is also sufficient.

\section{ACKNOWLEDGMENTS}

The author would like to thank Vladimir Kadets, Javier Merí, Rafael Payá, and Dirk Werner for fruitful conversations concerning the matter of this paper. He also thanks the anonymous referee for productive comments on the subject of the paper.

\section{REFERENCES}

[1] Y. Abramovich and C. Aliprantis, An Invitation to Operator Theory, Graduate Texts in Math. 50, Amer. Math. Soc., Providence, RI, 2002. MR.1921782 (2003h:47072)

[2] Y. Abramovich and C. Aliprantis, Problems in Operator Theory, Graduate Texts in Math. 51, Amer. Math. Soc., Providence, RI, 2002. MR1921783 (2003h:47073)

[3] F. Albiac and N. J. Kalton, Topics in Banach Space Theory, Graduate Texts in Mathematics 233, Springer-Verlag, New York, 2006. MR2192298 (2006h:46005)

[4] J. Becerra, G. López, A. M. Peralta, and A. Rodríguez-Palacios, Relatively weakly open sets in closed balls of Banach spaces and real $J B^{*}$-triples of finite rank, Math. Ann. 330 (2004), 45-58. MR2091678 (2005f:46128)

[5] J. Becerra Guerrero And M. Martín, The Daugavet property of $C^{*}$-algebras, J $B^{*}$ triples, and of their isometric preduals, J. Funct. Anal. 224 (2005), 316-337. MR.2146042 (2006g:46009)

[6] F. F. Bonsall and J. Duncan, Numerical Ranges of Operators on Normed Spaces and of Elements of Normed Algebras, London Math. Soc. Lecture Note Ser. 2, Cambridge University Press, London-New York, 1971. MR0288583 (44:5779)

[7] F. F. Bonsall and J. Duncan, Numerical Ranges. II, London Math. Soc. Lecture Note Ser. 10, Cambridge University Press, London-New York, 1973. MR 0442682 (56:1063)

[8] K. Boyko, V. Kadets, M. Martín, and D. Werner, Numerical index of Banach spaces and duality, Math. Proc. Cambridge Phil. Soc. 142 (2007), 93-102. MR2296393 
[9] K. Boyko, V. Kadets, M. Martín, and J. Merí, Properties of lush spaces and applications to Banach spaces with numerical index 1, Studia Math. 190 (2009), 117-133.

[10] J. Duncan, C. M. McGregor, J. D. Pryce, and A. J. White, The numerical index of a normed space, J. London Math. Soc. 2 (1970), 481-488. MR0264371 (41:8967)

[11] C. Finet, M. Martín, and R. Payá, Numerical index and renorming, Proc. Amer. Math. Soc. 131 (2003), 871-877. MR.1937425 (2003h:46021)

[12] Y. Friedman and B. Russo, Structure of the predual of a $J B W^{*}$-triple, J. Reine Angew. Math. 356 (1985), 67-89. MR779376 (86f:46073)

[13] P. Harmand, D. Werner, And W. Werner, M-ideals in Banach Spaces and Banach Algebras, Lecture Notes in Math. 1547, Springer-Verlag, Berlin, 1993. MR.1238713 (94k:46022)

[14] J. M. Isidro, W. Kaup, And Á. Rodríguez-Palacios, On real forms of $J B^{*}$-triples, Manuscripta Math. 86 (1995), 311-335. MR.1323795 (96a:46121)

[15] V. Kadets. M. Martín, and R. PAyÁ, Recent progress and open questions on the numerical index of Banach spaces, RACSAM Rev. R. Acad. Cienc. Exactas Fis. Nat. Serie A Mat. 100 (2006), 155-182. MR2267407 (2007h:46011)

[16] V. Kadets, R. Shvidkoy, G. Sirotkin, And D. Werner, Banach spaces with the Daugavet property, Trans. Amer. Math. Soc. 352 (2000), 855-873. MR1621757 (2000c:46023)

[17] A. Kaidi, A. Morales, and A. Rodriguez Palacios, Geometrical properties of the product of a $C^{*}$-algebra, Rocky Mountain J. Math. 31 (2001), 197-213. MR.1821377 (2002f:46140)

[18] G. López, M. Martín, and R. PayÁ, Real Banach spaces with numerical index 1, Bull. London Math. Soc. 31 (1999), 207-212. MR.1664125 (99k:46024)

[19] M. Martín, The alternative Daugavet property of $C^{*}$-algebras and $J B^{*}$-triples, Math. Nachr. 281 (2008), 376-385. MR2392119

[20] M. Martín and T. Oikhberg, An alternative Daugavet property, J. Math. Anal. Appl. 294 (2004), 158-180. MR2059797 (2005b:46023)

[21] M. Martín and R. PAyÁ, Numerical index of vector-valued function spaces, Studia Math. 142 (2000), 269-280. MR1792610 (2001i:46017)

[22] H. Pfitzner, Separable $L$-embedded Banach spaces are unique preduals, Bull. London Math. Soc. 39 (2007), 1039-1044. MR2392827 (2009a:46030)

[23] B. Russo, Structure of $J B^{*}$-triples, in: Jordan Algebras (Oberwolfach, 1992), 209-280, de Gruyter, Berlin, 1994. MR.1293321 (95h:46109)

[24] D. Werner, Recent progress on the Daugavet property, Irish Math. Soc. Bull. 46 (2001), 77-97. MR $1856978(2002 \mathrm{i}: 46014)$

Departamento de Análisis Matemático, Facultad de Ciencias, Universidad de Granada, E-18071 Granada, Spain

E-mail address: mmartins@ugr.es 